\title{
Asbestos-related lung cancers are rarely associated with ALK, ROS1 and RET rearrangements
}

\author{
To the Editor:
}

Lung cancer remains the main cause of cancer death. In addition to smoking, exposure to occupational carcinogens constitutes a major risk for lung cancer. Among occupational cancers, asbestos-related ones are the most frequent, accounting for about 5-7\% of lung cancers. A better understanding of the molecular pathways implicated in the progression of cancer has greatly improved the therapeutic management of patients with advanced lung cancers, especially nonsmall cell lung cancer (NSCLC) of the adenocarcinoma subtype. Indeed, several therapies targeting the epidermal growth factor receptor (EGFR) or anaplastic lymphoma kinase (ALK)/ROS1 pathways are now approved to treat patients with advanced EGFR-mutated or ALK/ROS1-rearranged NSCLC, respectively. While EGFR and ALK alterations are mainly encountered in NSCLC of nonsmokers, other biomarkers are more frequent in smoking patients, such as KRAS mutations, and are of prognostic interest. Nevertheless, the frequencies of the different biomarkers analysed daily for the therapeutic management of patients with NSCLC are rarely reported in occupational lung cancers, including asbestos-related ones.

The study by PARIS et al. [1] of the frequencies of EGFR, ALK, KRAS, HER2, BRAF and PIK3 molecular alterations in never-smokers with lung cancer and occupational exposure to carcinogens including asbestos is very interesting in this manner. However, others biomarker that are also of therapeutic interest were not studied by PARIs et al. [1], such as ROS1, RET, NTRK1 and MET. We have also just conducted a study focusing on oncogenic chromosomal rearrangements in a cohort of French patients with financially compensated asbestos-related lung cancers. Here, we intend to present the results of our monocentric retrospective study.

In a previous work, we reported the clinical, occupational and histopathological features of 146 patients with financially compensated asbestos-related lung cancers in our occupational disease centre between 2003 and 2013 [2]. As an extension of this study and after approval by our institutional review board (CHRU Brest 2016.CE10), we collected formalin-fixed, paraffin-embedded tumour samples to perform additional molecular analyses in 56 patients (for 90 patients, no tumour sample was available at the time of our retrospective study). We performed not only ALK analyses, as reported by PARIs et al. [1], using immunohistochemistry (5A4 clone; Clinisciences, Nanterre, France) and fluorescence in situ hybridisation (FISH) analyses (Vysis LSI ALK Dual Color Break Apart FISH probe; Abbott Molecular, Rungis, France) but also ROS1 analyses (immunohistochemistry with clone D4D6 (Cell Signaling Technology; Ozyme, Saint Quentin en Yvelines, France) and FISH with Vysis 6q22 ROS1 Break Apart FISH Probe Kit (Abbott Molecular)) as previously reported [3, 4]. Using FISH, we also searched for RET and neurotrophic NTRK1 rearrangements (probes ON RET (10q11) Break (Kreatech/Leica, Nanterre, France) and ZytoLight SPEC NTRK1 Dual Color Break Apart Probe (Zytovision GmbH, Bremerhaven, Germany)) as well as MET amplification (Vysis LSI D7S486 (7q31) SpectrumOrange/Vysis CEP 7 SpectrumGreen Probe, Abbott Molecular).

Among 56 tumours, 32 were adenocarcinomas, 20 were squamous cell carcinomas and four were small cell carcinomas. Major differences in comparison with the study by PARIS et al. [1] were that all patients were men and that nonsmokers only represented five (9\%) patients in our cohort. Oncogenic chromosomal rearrangements were encountered in lung adenocarcinomas of four (7\%) patients: two ALK-rearranged

@ERSpublications

$A L K, R O S 1$ and RET rearrangements can also be diagnosed in asbestos-related lung cancers http://ow.ly/rFpb30hV5Wt

Cite this article as: Uguen $\mathrm{M}$, Dewitte J-D, Loddé B, et al. Asbestos-related lung cancers are rarely associated with ALK, ROS1 and RET rearrangements. Eur Respir J 2018; 51: 1702605 [https://doi.org/ 10.1183/13993003.02605-2017]. 
tumours (with positive immunohistochemistry and FISH results) in a never-smoker and a 20-pack-year smoker, one ROS1-rearranged tumour in a 10-pack-year smoker (with positive immunohistochemistry and FISH results), and one RET-rearranged tumour in a 20-pack-year smoker (positive FISH result). No NTRK1 rearrangement or MET amplification was diagnosed.

Tobacco and asbestos are known to have synergistic carcinogenic effects, with 10- and five-fold increased risks of lung cancer, respectively, if considered separately, and a 50-fold increased risk in patients with both exposures. Albeit that oncogenic rearrangements are classically described in NSCLC of young and never-smoking patients, we regularly diagnose $A L K$ rearrangements in patients with a smoking history [4]. Asbestos is known to cause recurrent chromosomal aberrations in lung cancers (e.g. in the 2p16, 9q33.1 and 19p13 loci) [5]. Beyond chromosomal gains and losses, chromosomal alterations leading to gene fusions or amplifications that are of therapeutic relevance have been detected in few ( $A L K, 2 \mathrm{p} 23$ locus) to no studied (ROS1, 6q22.1 locus; RET, 10q11.21 locus; NTRK1, 1q23.1 locus; C-MET, 7q31.2 locus) in asbestos-related lung cancers. In the study by PARIS et al. [1], none of the 21 asbestos-exposed patients had an $A L K$-rearranged cancer [1]. In our study, we have demonstrated that chromosomal alterations of theranostic relevance are rare but can be encountered among patients with asbestos-related lung cancers, among smokers and nonsmokers, opening potential access to targeted therapies for these patients.

In our study, having considered only patients with financially compensated asbestos-related lung cancers constitutes a bias preventing any estimation of the frequency of chromosomal rearrangements among asbestos-related lung cancers. In a future work, it would be interesting (and may be feasible in the cohort of PARIs et al. [1]) to analyse also ROS1, RET, NTRK1 and MET in a larger cohort of asbestos-exposed never-smokers to further study the relationship between asbestos exposure and chromosomal aberrations of theranostic interest.

Marie Uguen ${ }^{1}$, Jean-Dominique Dewitte ${ }^{2,3}$, Brice Loddé ${ }^{2,3}$, Pascale Marcorelles ${ }^{3,4}$ and Arnaud Uguen $\oplus^{3,4,5}$

${ }^{1}$ Santé au Travail en Iroise, Brest, France. ${ }^{2}$ CHRU Brest, Dept of Occupational and Environmental Diseases, Brest, France. ${ }^{3}$ European University of Brittany, Brest, France. ${ }^{4} \mathrm{CHRU}$ Brest, Dept of Pathology, Brest, France. ${ }^{5}$ Inserm, U1078, Brest, France.

Correspondence: Arnaud Uguen, Dept of Pathology, University Hospital Morvan, 5, Avenue Foch 29609 Brest, France. E-mail: arnaud.uguen@chu-brest.fr

Received: Dec 152017 | Accepted after revision: Dec 302017

Support statement: This study was supported by La Ligue contre le cancer CD29. Funding information for this article has been deposited with the Crossref Funder Registry.

Conflict of interest: None declared.

Acknowledgements: We would like to also acknowledge the pathologists of Brest and the Local tumor tissue biobank BB-0033-00037 (“CRB Santé/Tumorothèque de Brest”) for their collaboration in this study and Miss Sandrine Duigou, Mrs Le Prunennec Annette and Mr Eric Gobin for technical assistance.

\section{References}

1 Paris C, Do P, Mastroianni B, et al. Association between lung cancer somatic mutations and occupational exposure in never-smokers. Eur Respir J 2017; 50: 1700716.

2 Uguen M, Dewitte JD, Marcorelles P, et al. Asbestos-related lung cancers: a retrospective clinical and pathological study. Mol Clin Oncol 2017; 7: 135-139.

3 Uguen A, Schick U, Quéré G. A rare case of ROS1 and ALK double rearranged non-small cell lung cancer. J Thorac Oncol 2017; 12: e71-e72.

4 Uguen A, Andrieu-Key S, Vergne F, et al. ALK ambiguous-positive non-small cell lung cancers are tumors challenged by diagnostic and therapeutic issues. Oncol Rep 2016; 36: 1427-1434.

5 Nymark P, Catalán J, Suhonen S, et al. Accumulation of genomic alterations in 2p16, 9q33.1 and 19p13 in lung tumours of asbestos-exposed patients. Mol Oncol 2013; 7: 29-40. 